\title{
Case Reports and Studies
}

Kirk R*

School of Health Sciences, University of Canterbury, New Zealand

${ }^{*}$ Corresponding author: Kirk R, Associate Professor, School of Health Sciences, University of Canterbury, New Zealand; Tel: 643364 3108, E-mail: ray.kirk@canterbury.ac.nz

Citation: Kirk R (2013) Case Reports and Studies. J Case Rep Stud 1(1): e101. doi: 10.15744/2348-9820.e101

Received Date: June 25, 2013 Accepted Date: July 29, 2013 Published Date: August 01, 2013

Welcome to the first issue of the Journal of Case Reports and Studies. Within the field of medicine a number of systems have been established to stratify the quality of research designs which have been collectively termed "levels of evidence" or a "hierarchy of evidence" approach. These approaches rank study designs with respect to their impact on internal and external validity. Typically case study designs are ranked near the bottom of these "evidence hierarchies". For example, the Centre for Evidence based medicine at Oxford university ranks case series on level 5 of a 1-6 level scale [1]. The gold standard in evidence based medicine is the randomised controlled trial (RCT) but most patients are excluded or ineligible to participate in RCTs [2]. It must be noted that these "levels of evidence" approaches are designed for research investigating the outcomes of clinical interventions or diagnostic procedures. The applicability of case reports and case studies goes beyond such research. What do we mean by case reports and case studies?

The American National Cancer Institute [3] defines a case report as "a detailed report of the diagnosis, treatment, and follow-up of an individual patient. Case reports also contain some demographic information about the patient (for example, age, gender, ethnic origin)" and defined a case series [4] as "a group or series of case reports involving patients who were given similar treatment. Reports of case series usually contain detailed information about the individual patients. This includes demographic information (for example, age, gender, ethnic origin) and information on diagnosis, treatment, response to treatment, and follow-up after treatment".

Case series can be designed to include all eligible participants under investigation (consecutive) or a selection of all elgible participants under investigation (non-consecutive). Case study methods can be either prospective where participants are followed over time, or retrospective where historical data is collected on the participants under investigation.

Volkland and Iles [5] noted that in medicine most case reports are on one of following six topics.

1. "An unexpected association between diseases or symptoms.

2. An unexpected event in the course of observing or treating a patient.

3. Findings that shed new light on the possible pathogenesis of a disease or an adverse effect.

4. Unique or rare features of a disease.

5. Unique therapeutic approaches.

6. A positional or quantitative variation of the anatomical structures."

Another type of case study design reported mostly in health and education is the " $n$-of- 1 " trial or "single subject" study design [6]. In medicine, a N-of-1 trial is a crossover trial, which may include randomization and often blinding to improve internal validity, and is applied to a single patient.

The journal editors look forward to receiving your manuscripts that will add to the knowledge base and advance the field of case report and case study research.

\section{References}

1. (2013) Oxford Centre for Evidence-based Medicine - Levels of Evidence (March 2009).

2. Guyatt GH, Haynes RB, Jaeschke RZ, Cook DJ, Green L, et al. (2000) Users' Guides to the Medical Literature: XXV. Evidence-based medicine: principles for applying the Users' Guides to patient care. Evidence-Based Medicine Working Group. JAMA 284: 1290-6.

3. (2010) Case report. NCI Dictionary of Cancer Term. National Cancer Institute (NCI).

4. (2010) Case series. NCI Dictionary of Cancer Term. National Cancer Institute (NCI).

5. Iles Robert L (1997) Guidebook to better medical writing. Island Press, Washington, DC.

6. Perdices M, Tate RL (2009) Single-subject designs as a tool for evidencebased clinical practice: Are they unrecognised and undervalued? Neuropsychol Rehabil 19: 904-27. 


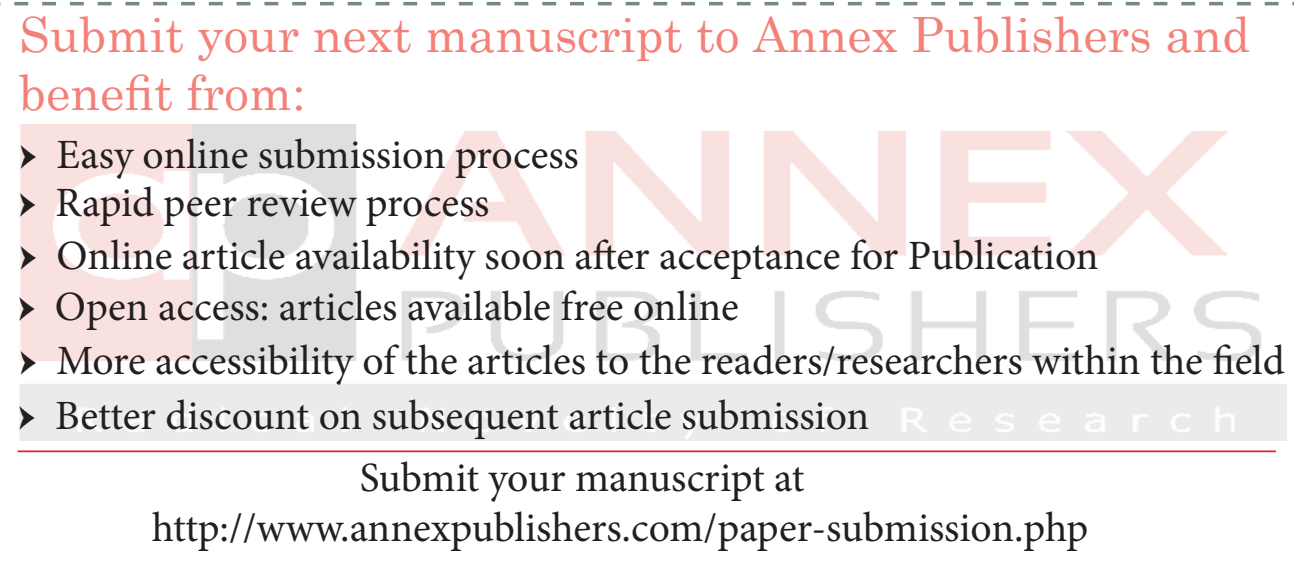

